\title{
Lattice Boltzmann Simulation of the Trapping of a Microdroplet in a Well of Surface Energy
}

\author{
Haihu Liu ${ }^{\mathrm{a}, *}$, Yonghao Zhang ${ }^{\mathrm{b}}$ \\ ${ }^{a}$ School of Energy and Power Engineering, Xi'an Jiaotong University, 28 West Xianning \\ Road, Xi'an 710049, China \\ ${ }^{b}$ James Weir Fluids Laboratory, Department of Mechanical $\&$ Aerospace Engineering, \\ University of Strathclyde, Glasgow G1 1 XJ, UK
}

\begin{abstract}
In this paper, a three-dimensional phase-field lattice Boltzmann method is used to simulate the dynamical behavior of a droplet, subject to an outer viscous flow, in a microchannel that contains a cylindrical hole etched into its top surface. The influence of the capillary number and the hole diameter (expressed as the ratio of hole diameter to channel height, $b$ ) is investigated. We demonstrate numerically that the surface energy gradient induced by the hole can create an anchoring force to resist the hydrodynamic drag from the outer flow, resulting in the droplet anchored to the hole when the capillary number is below a critical value. As $b$ increases, the droplet can be anchored more easily. For $b<2$, the droplet partially enters into the hole and forms a spherical cap; whereas for $b>2$, the spherical cap of droplet reaches the top wall of the hole, making the hole depth into an additional important parameter. These observations are consistent with the previously reported experiments. However, the droplet does not fully fill the hole for $b>2$, departing from the expectation of Dangla et al. [R. Dangla, S. Lee, C. N. Baroud, Trapping microfluidic drops in wells of surface energy, Phys. Rev. Lett. 107 (2011) 124501]. Also, it is observed in the anchored state that the rear of the droplet rests at a small distance away from the junction. Finally, the droplet undergoes a slow-down process only when its
\end{abstract}

\footnotetext{
* Corresponding author

Email address: haihu.liu@xjtu.edu.cn (Haihu Liu)
} 
rear passes through the hole, regardless of $b$.

Keywords: Droplet manipulation, Microfluidics, Surface energy gradient, Surface wettability, Lattice Boltzmann method

\section{Introduction}

Droplet-based microfluidics has recently emerged as a new and exciting technological platform for chemical, biological and pharmaceutical processes and analyses. Manipulation of droplets in a precise and flexible manner plays a vital role in these applications. A number of approaches have been commonly used to manipulate the dynamical behavior of droplets in microfluidics, including electrowetting on dielectric (EWOD) [1, 2], dielectrophoresis (DEP) [3, 4], hydrodynamic stress $[5,6,7]$, thermocapillary force $[8,9,10,11]$, surface acoustic wave [12], magnetic force $[13,14]$, and optical forces $[15,16]$. In recent years, a novel use of surface energy gradients was demonstrated to guide or anchor droplets against a mean flow in a confined microchannel [17, 18]. The surface energy gradient can be created by modulations of the channel height, e.g., by etching a pattern of holes and grooves into the top surface of the channels. Compared to commonly-used approaches, the use of surface energy gradients for microdroplet manipulation has many advantages, such as simple operation and high efficiency, and ease of parallelization that enables a high-throughput production of droplet arrays for chemical or biomedical studies. Besides, it also allows the use of continuous flow to control the chemical environment and content of the trapped droplets, in both time and space.

The trapping mechanism of a confined droplet subject to a surface energy gradient induced by a hole has been discussed in [18]. From a viewpoint of free energy, a droplet tends to evolve towards the direction of decreasing its surface free energy, which is equal to its surface area times the interfacial tension. Since the hole allows the droplet to reduce its surface area and thus the surface energy, the droplet resists leaving it into a more squeezed region. The resistance force is given by the surface energy gradient, which acts as an anchoring force 
to block the droplet movement [18]. On the other hand, the drag force, exerted by the outer flow, tends to push the droplet out of the hole. As a result, the relative magnitude between the anchoring and drag forces determines whether the droplet is anchored or not. Based on the balance between the two forces, an analytical expression was derived to predict the shape of anchored droplet [18]. In this derivation, it is assumed that the droplet fluid completely repels the walls and the ratio of hole diameter to channel height should be not more than 2. These assumptions will significantly restrict the usefulness of the analytical formulae because various materials can be used to fabricate the channels with different dimensions. Numerical modelling and simulations can complement theoretical and experimental studies, allowing us to visualize the transient flowfiled and accurately quantify the interface structures for both anchored and unanchored/released droplets. In addition, they are of potential to be extended to multiple droplets or complex geometries for prospective applications such as rails and anchors in [17]. However, it is challenging to use traditional computational fluid dynamics (CFD) methods, e.g., the volume-of-fluid (VOF) [19, 20] and level-set (LS) methods [21, 22], for simulating the dynamical behavior of a confined droplet in a microchannel because of the difficulties in modelling and capturing the dynamic phase interfaces [23]. Also, minimization of unphysical spurious currents at the interface still remains a major challenge for these methods. In addition, a suitable slip model with slip length at the molecular scale has to be introduced to avoid stress singularities at the moving contact line. Microscopically, the interface between different phases and the contact-line dynamics on the solid surface are due to interparticle interactions [24]. Thus, mesoscopic level models are expected to accurately describe the microdroplet dynamics in a confined microchannel.

The lattice Boltzmann method (LBM) has been developed into a promising numerical tool for simulating complex fluid flows. Unlike the traditional CFD methods, the LBM is built upon the mesoscopic kinetic equation for particle distribution functions. Due to its kinetic nature, the LBM has been found to be particularly useful for the simulation of multiphase flows. In the LBM 
community, a number of multiphase models have been proposed, and they can be generally classified into four types, i.e., the color-fluid model $[25,26]$, the interparticle-potential model $[27,28]$, the free-energy model $[29,30]$, and the phase-field model [31, 32, 33, 34]. For a comprehensive review of these models, interested readers may refer to [23]. In this paper, a three-dimensional (3D) phase-field LBM is used to simulate the dynamical behavior of a confined droplet, subject to an external viscous flow, in a microchannel that contains a cylindrical hole etched on the top surface. In this method, the spurious currents are effectively suppressed by the use of an interfacial tension force of potential form and the multiple-relaxation-time (MRT) model for fluid flow; the contact-line dynamics is modeled by the method of Briant et al. [35], with simple implementation for complex solid surface following Niu et al. [36]. We show how the anchor blocks the droplet motion against an external flow and investigate the influence of capillary number and hole diameter (expressed as the ratio of hole diameter to channel height, $b$ ) on the droplet motion. In this study, the walls are not limited to perfect hydrophobicity to the droplet (i.e. the contact angle of the droplet on the solid walls is not necessarily equal to 180 degrees), and the ratio of hole diameter to channel height is not limited to the case of $b \leq 2$, thus complementing the previous study by Dangla et al. [18] and enhancing our understanding of the trapping behavior of a droplet in surface energy wells.

\section{Numerical Method}

\subsection{Phase-field theory}

We consider here a phase-field lattice Boltzmann model for incompressible immiscible two-phase flows. Suppose that there are two incompressible immiscible fluids, say oil and water. The order parameter $\phi$ is utilized to identify different fluids and is assumed to be constant in the bulk fluids, e.g. $\phi=-1$ for the bulk oil while $\phi=1$ for the bulk water. Across the interfacial region, there is a rapid but smooth change of $\phi$. The time evolution of the diffuse interface 
is governed by the Cahn-Hilliard equation (CHE) [37]

$$
\partial_{t} \phi+\vec{u} \cdot \nabla \phi=M \nabla^{2} \mu,
$$

where $t$ is the time, $\vec{u}$ is the fluid velocity, and $M$ is the mobility. The chemical potential $\mu$ in Eq.(1) can be derived from the free-energy functional

$$
\Psi=\int_{V} \psi(\phi, \nabla \phi) d V=\int_{V}\left[\frac{A}{4}\left(1-\phi^{2}\right)^{2}+\frac{\kappa}{2}|\nabla \phi|^{2}\right] d V,
$$

where $A$ and $\kappa$ are the parameters related to the interfacial tension and the interface thickness. By minimizing the free energy functional, the chemical potential is given by

$$
\mu=\frac{\delta \psi}{\delta \phi}=A \phi\left(\phi^{2}-1\right)-\kappa \nabla^{2} \phi
$$

For a planar oil-water interface in a quiescent infinite system, the order parameter profile across the interface can be obtained from Eq.(3) at $\mu=0$,

$$
\phi(x)=\tanh (x / \xi)
$$

where $x$ is the spatial location normal to the interface $(x=0)$, and $\xi$ is a measure of the interface thickness, which is defined as $\xi=\sqrt{\frac{2 \kappa}{A}}$. The interfacial tension $\sigma$ can be interpreted as the excess free energy per unit interface area, and for a planar interface in equilibrium, it can be evaluated by

$$
\sigma=\int_{-\infty}^{\infty} \kappa|\nabla \phi|^{2} d x=\frac{4 \kappa}{3 \xi}
$$

With consideration of a constant interfacial tension, the Navier-Stokes equations (NSEs) for the incompressible fluid flows can be written as [11]

$$
\begin{gathered}
\nabla \cdot \vec{u}=0, \\
\rho\left(\partial_{t} \vec{u}+\vec{u} \cdot \nabla \vec{u}\right)=-\nabla p+\mu \nabla \phi+\nabla \cdot\left[\eta\left(\nabla \vec{u}+\nabla \vec{u}^{T}\right)\right],
\end{gathered}
$$

where $p$ is the pressure, and $\rho$ and $\eta$ are the density and the viscosity of fluid mixture. In a typical oil-water microfluidic system, the Bond number that characterizes the relative importance of gravity and interfacial tension, is so 
small that the effect of the density difference can be ignored. It is therefore assumed, for simplicity, that both fluids have equal densities, which are given by $\rho_{0}$. Note that the interfacial tension force in Eq.(7) is expressed as a potential form, which produces much smaller spurious currents than its counterpart of pressure form $[38,39,11]$.

When the fluid-surface interactions are taken into account, the wetting condition proposed by Briant et al. [35] is imposed at the solid wall, which provides the boundary condition for the order parameter $\phi$,

$$
\left.\vec{n} \cdot \nabla \phi\right|_{w}=-\Theta \sqrt{A / 2 \kappa}
$$

where $\vec{n}$ is the local normal direction of the wall pointing into the fluid, and $\Theta$ is the wetting potential, which is related to the contact angle $\theta$ by

$$
\cos (\theta)=\frac{(1+\Theta)^{3 / 2}-(1-\Theta)^{3 / 2}}{2}
$$

\subsection{Lattice Boltzmann method}

The NSEs and the CHE are solved in a LBM framework. Specifically, the NSEs are solved using the MRT model, while the CHE is solved through the standard Bhatnagar-Gross-Krook (BGK) model. Two particle distribution functions (PDFs) $f_{i}(\vec{x}, t)$ and $g_{i}(\vec{x}, t)$ are employed on each lattice site, where $i$ is the lattice direction. The first distribution function is related to the macroscopic density $\rho$ and the momentum $\vec{j}$, and the second distribution function captures the order parameter $\phi$

$$
\rho(\vec{x}, t)=\sum_{i} f_{i}(\vec{x}, t), \vec{j}(\vec{x}, t)=\sum_{i} f_{i}(\vec{x}, t) \vec{e}_{i}+\frac{1}{2} \mu \nabla \phi \delta_{t}, \phi(\vec{x}, t)=\sum_{i} g_{i}(\vec{x}, t),
$$

where $\vec{j}=\rho_{0} \vec{u}, \rho_{0}$ is used instead of $\rho$ to reduce compressibility effects in the model $[40,41]$, and $\vec{e}_{i}$ is the lattice velocity vector defined as

$$
\vec{e}_{i}= \begin{cases}(0,0,0), & i=0 \\ ( \pm 1,0,0) c,(0, \pm 1,0) c,(0,0, \pm 1) c, & i=1,2, \cdots, 6 \\ ( \pm 1, \pm, 0) c,( \pm 1,0, \pm 1) c,(0, \pm 1, \pm 1) c, & i=7,8, \cdots, 18\end{cases}
$$


for the 3D 19-velocity model (D3Q19). The lattice speed $c$ is defined by $c=$ $\delta_{x} / \delta_{t}$, where $\delta_{x}$ and $\delta_{t}$ are the lattice spacing and time step, respectively. The speed of sound $c_{s}$ is related to $c$ by $c_{s}=c / \sqrt{3}$.

The evolution equations of $f_{i}(\vec{x}, t)$ and $g_{i}(\vec{x}, t)$ can be written as [11]

$$
\begin{gathered}
f_{i}\left(\vec{x}+\vec{e}_{i} \delta_{t}, t+\delta_{t}\right)-f_{i}(\vec{x}, t)=-\left(\mathbf{M}^{-1} \mathbf{S M}\right)_{i j}\left[f_{j}(\vec{x}, t)-f_{j}^{e q}(\vec{x}, t)\right]+\delta_{t} \bar{F}_{i}, \\
g_{i}\left(\vec{x}+\vec{e}_{i} \delta_{t}, t+\delta_{t}\right)-g_{i}(\vec{x}, t)=-\frac{1}{\tau_{g}}\left[g_{i}(\vec{x}, t)-g_{i}^{e q}(\vec{x}, t)\right],
\end{gathered}
$$

where $f_{i}^{e q}$ and $g_{i}^{e q}$ are the equilibrium distribution functions of $f_{i}$ and $g_{i}, \tau_{g}$ is the single relaxation parameter for $g_{i}, \mathbf{M}$ is a transformation matrix, $\mathbf{S}$ is a diagonal relaxation matrix, and $\bar{F}_{i}$ represents the discrete forcing term accounting for the interfacial tension force acting on the fluid mixture, which is given by

$$
\overline{\mathbf{F}}=\mathbf{M}^{-1}\left(\mathbf{I}-\frac{1}{2} \mathbf{S}\right) \mathbf{M} \tilde{\mathbf{F}}
$$

where $\mathbf{I}$ is a unit matrix, $\overline{\mathbf{F}}=\left[\bar{F}_{0}, \bar{F}_{1}, \bar{F}_{2}, \ldots, \bar{F}_{18}\right]^{T}$, and $\tilde{\mathbf{F}}=\left[\tilde{F}_{0}, \tilde{F}_{1}, \tilde{F}_{2}, \ldots, \tilde{F}_{18}\right]^{T}$.

The governing physics of LBM is determined through the hydrodynamic moments of the equilibrium distribution functions and the forcing terms. The moments of $f_{i}^{e q}, g_{i}^{e q}$ and $\tilde{F}_{i}$ are:

$$
\begin{gathered}
\sum_{i} f_{i}^{e q}=\rho, \quad \sum_{i} f_{i}^{e q} e_{i \alpha}=\rho_{0} u_{\alpha}, \quad \sum_{i} f_{i}^{e q} e_{i \alpha} e_{i \beta}=\rho_{0} u_{\alpha} u_{\beta}+\rho c_{s}^{2} \delta_{\alpha \beta}, \\
\sum_{i} f_{i}^{e q} e_{i \alpha} e_{i \beta} e_{i \gamma}=\rho_{0} c_{s}^{2}\left(\delta_{\alpha \beta} u_{\gamma}+\delta_{\alpha \gamma} u_{\beta}+\delta_{\beta \gamma} u_{\alpha}\right), \\
\sum_{i} g_{i}^{e q}=\phi, \quad \sum_{i} g_{i}^{e q} e_{i \alpha}=\phi u_{\alpha}, \quad \sum_{i} g_{i}^{e q} e_{i \alpha} e_{i \beta}=\phi u_{\alpha} u_{\beta}+\Gamma \mu \delta_{\alpha \beta}, \\
\sum_{i} \tilde{F}_{i}=0, \quad \sum_{i} \tilde{F}_{i} e_{i \alpha}=F_{S \alpha}, \quad \sum_{i} \tilde{F}_{i} e_{i \alpha} e_{i \beta}=u_{\alpha} F_{S \beta}+u_{\beta} F_{S \alpha} .
\end{gathered}
$$

By satisfying these moments, $f_{i}^{e q}, g_{i}^{e q}$ and $\tilde{F}_{i}$ can be chosen as

$$
\begin{aligned}
f_{i}^{e q} & =w_{i}\left[\rho+\rho_{0}\left(\frac{\vec{e}_{i} \cdot \vec{u}}{c_{s}^{2}}+\frac{\left(\vec{e}_{i} \cdot \vec{u}\right)^{2}}{2 c_{s}^{4}}-\frac{|\vec{u}|^{2}}{2 c_{s}^{2}}\right)\right], \\
g_{i}^{e q} & =w_{i}\left[G_{i}+\phi\left(\frac{\vec{e}_{i} \cdot \vec{u}}{c_{s}^{2}}+\frac{\left(\vec{e}_{i} \cdot \vec{u}\right)^{2}}{2 c_{s}^{4}}-\frac{|\vec{u}|^{2}}{2 c_{s}^{2}}\right)\right], \\
\tilde{F}_{i} & =w_{i}\left[\frac{\vec{e}_{i}-\vec{u}}{c_{s}^{2}}+\frac{\left(\vec{e}_{i} \cdot \vec{u}\right) \vec{e}_{i}}{c_{s}^{4}}\right] \cdot \mu \nabla \phi,
\end{aligned}
$$

where the coefficient $G_{i}$ is given by

$$
G_{i}= \begin{cases}\Gamma \mu / c_{s}^{2} & (i>0) \\ {\left[\phi-\left(1-w_{0}\right) \Gamma \mu / c_{s}^{2}\right] / w_{0}} & (i=0)\end{cases}
$$


and $w_{i}$ is the weight factor with $w_{0}=1 / 3, w_{1-6}=1 / 18$ and $w_{7-18}=1 / 36$.

The transformation matrix $\mathbf{M}$ is designed to contain more physically relevant quantities, e.g. density, momentum, energy, and their fluxes, and is explicitly given by

$$
\mathbf{M}=\left(\begin{array}{ccccccccccccccccccc}
1 & 1 & 1 & 1 & 1 & 1 & 1 & 1 & 1 & 1 & 1 & 1 & 1 & 1 & 1 & 1 & 1 & 1 & 1 \\
-30 & -11 & -11 & -11 & -11 & -11 & -11 & 8 & 8 & 8 & 8 & 8 & 8 & 8 & 8 & 8 & 8 & 8 & 8 \\
12 & -4 & -4 & -4 & -4 & -4 & -4 & 1 & 1 & 1 & 1 & 1 & 1 & 1 & 1 & 1 & 1 & 1 & 1 \\
0 & 1 & -1 & 0 & 0 & 0 & 0 & 1 & -1 & 1 & -1 & 1 & -1 & 1 & -1 & 0 & 0 & 0 & 0 \\
0 & -4 & 4 & 0 & 0 & 0 & 0 & 1 & -1 & 1 & -1 & 1 & -1 & 1 & -1 & 0 & 0 & 0 & 0 \\
0 & 0 & 0 & 1 & -1 & 0 & 0 & 1 & 1 & -1 & -1 & 0 & 0 & 0 & 0 & 1 & -1 & 1 & -1 \\
0 & 0 & 0 & -4 & 4 & 0 & 0 & 1 & 1 & -1 & -1 & 0 & 0 & 0 & 0 & 1 & -1 & 1 & -1 \\
0 & 0 & 0 & 0 & 0 & 1 & -1 & 0 & 0 & 0 & 0 & 1 & 1 & -1 & -1 & 1 & 1 & -1 & -1 \\
0 & 0 & 0 & 0 & 0 & -4 & 4 & 0 & 0 & 0 & 0 & 1 & 1 & -1 & -1 & 1 & 1 & -1 & -1 \\
0 & 2 & 2 & -1 & -1 & -1 & -1 & 1 & 1 & 1 & 1 & 1 & 1 & 1 & 1 & -2 & -2 & -2 & -2 \\
0 & -4 & -4 & 2 & 2 & 2 & 2 & 1 & 1 & 1 & 1 & 1 & 1 & 1 & 1 & -2 & -2 & -2 & -2 \\
0 & 0 & 0 & 1 & 1 & -1 & -1 & 1 & 1 & 1 & 1 & -1 & -1 & -1 & -1 & 0 & 0 & 0 & 0 \\
0 & 0 & 0 & -2 & -2 & 2 & 2 & 1 & 1 & 1 & 1 & -1 & -1 & -1 & -1 & 0 & 0 & 0 & 0 \\
0 & 0 & 0 & 0 & 0 & 0 & 0 & 1 & -1 & -1 & 1 & 0 & 0 & 0 & 0 & 0 & 0 & 0 & 0 \\
0 & 0 & 0 & 0 & 0 & 0 & 0 & 0 & 0 & 0 & 0 & 0 & 0 & 0 & 0 & 1 & -1 & -1 & 1 \\
0 & 0 & 0 & 0 & 0 & 0 & 0 & 0 & 0 & 0 & 0 & 1 & -1 & -1 & 1 & 0 & 0 & 0 & 0 \\
0 & 0 & 0 & 0 & 0 & 0 & 0 & 1 & -1 & 1 & -1 & -1 & 1 & -1 & 1 & 0 & 0 & 0 & 0 \\
0 & 0 & 0 & 0 & 0 & 0 & 0 & -1 & -1 & 1 & 1 & 0 & 0 & 0 & 0 & 1 & -1 & 1 & -1 \\
0 & 0 & 0 & 0 & 0 & 0 & 0 & 0 & 0 & 0 & 0 & 1 & 1 & -1 & -1 & -1 & -1 & 1 & 1
\end{array}\right) .
$$

The diagonal relaxation matrix $\mathbf{S}$ is simply taken as

$$
\mathbf{S}=\operatorname{diag}[0,1,1,0,1,0,1,1,1, \omega, 1, \omega, 1, \omega, \omega, \omega, 1,1,1],
$$

where $\omega=1 / \tau_{f}$ which is related to the kinematic viscosity by $\nu=\left(\tau_{f}-0.5\right) c_{s}^{2} \delta_{t}$. It was demonstrated by Pooley et al. [42] that the choice of Eq.(23) can greatly suppress unphysical spurious currents in the vicinity of the contact line, avoiding generation of incorrect contact angle in BGK LBM simulations. Note that the use of MRT for reducing spurious currents at interfaces was also found in other multiphase LBMs $[43,44,11]$.

Using the Chapman-Enskog multiscale expansion, Eqs. (12) and (13) can lead to the hydrodynamic equations (6), (7) and (1) with the pressure $p=\rho c_{s}^{2}$ in the limit of the low Mach number. The relaxation parameter $\tau_{g}$ is related to the mobility through [29]

$$
M=\Gamma\left(\tau_{g}-0.5\right) \delta_{t},
$$

where $\Gamma$ is a constant that appears in the equilibrium distribution function $g_{i}^{e q}$. Note that it is not necessary to adopt a MRT model for $g_{i}$ since one can simply set $\tau_{g}=1$ and independently use $\Gamma$ to control the mobility [42]. 


\subsection{Boundary conditions}

No-slip boundary condition is applied for all the solid walls by the halfway bounce-back rule, which conveniently resolves the complex wall shapes and prevents leakage of the fluid mass across the walls $[45,46]$. The constant velocity and pressure boundary conditions are imposed at the inlet and outlet by the bounce-back of the non-equilibrium distribution rule developed by Zou and He [47]. We assume that fluids are only one pure single-component at inlet or outlet, where the unknown $g_{i}$ can be determined by the use of the method proposed by Hao and Cheng [48].

The wetting boundary condition at the solid wall can be implemented following the method proposed by Niu et al. [36], which is capable of dealing with complex solid boundaries with ease. In their method, the derivative of order parameter in Eq.(8) is evaluated by the first-order finite difference as $\partial_{n} \phi=\left(\phi_{f}-\phi_{s}\right) / \delta_{x}$, in which $\phi_{s}$ is the order parameter of the solid node and $\phi_{f}$ is the order parameter of fluid nodes adjacent to the solid node. By substituting the finite differences into Eq.(8) and averaging them over all fluid nodes adjacent to the solid node, the order parameter $\phi_{s}$ can be approximated by

$$
\phi_{s}=\frac{1}{N} \sum_{N}\left(\phi_{f}+\sqrt{\frac{A}{2 \kappa}} \Theta \delta_{x}\right),
$$

where $N$ is the total number of the fluid nodes which are nearest to the targeted solid node. It is worth noting that the present model is a diffuse-interface model with finite interface thickness. Although a no-slip boundary condition is used at the solid walls, the motion of contact lines arises naturally as a result of the diffusive flow that occurs in the diffuse interface region. Therefore, it is not surprising that all of the phase-field LBM models have used no-slip boundary condition at the solid walls for contact-line motion, see, e.g., Refs. [36, 48, 33, 49].

Once $\phi_{s}$ is determined, the gradient and Laplacian operators appearing in Eqs.(3) and (20) can be evaluated using 19-point finite difference stencils as 
follows:

$$
\begin{aligned}
\nabla \phi(\vec{x}) & =\frac{1}{c_{s}^{2} \delta_{t}} \sum_{i} w_{i} \phi\left(\vec{x}+\vec{e}_{i} \delta_{t}\right) \vec{e}_{i} \\
\nabla^{2} \phi(\vec{x}) & =\frac{2}{c_{s}^{2} \delta_{t}} \sum_{i} w_{i}\left[\phi\left(\vec{x}+\vec{e}_{i} \delta_{t}\right)-\phi(\vec{x})\right],
\end{aligned}
$$

which help to enhance the stability and accuracy of numerical model.

\section{Results and discussion}

In this section, we use the phase-field LBM to simulate the dynamical behavior of a confined water droplet, subject to an external flow of oil, in a microchannel that contains a cylindrical hole (i.e., anchor) of diameter between $d=60$ and $112 \mu \mathrm{m}$ and of depth $e=32 \mu \mathrm{m}$, as sketched in Fig.1. The channel has a length of $l=862 \mu \mathrm{m}$ in the $x$-direction and a width of $w=1000 \mu \mathrm{m}$ in the $y$-direction. The channel height is uniform except the part occupied by the hole, which is $h=40 \mu \mathrm{m}$. A cylindrical droplet of radius $R=160 \mu \mathrm{m}$ is initially centered at $x_{0}=y_{0}=250 \mu \mathrm{m}$. All boundaries except the inlet and outlet are considered as stationary walls with no-slip condition. The continuous phase oil is injected continuously from the inlet at a flow rate $Q_{c}$, which is a constant in each of the cases studied.

As indicated by a recent study [18], the migration of a confined droplet in the surface energy gradients, which are induced by the etched hole, can be characterized by three important dimensionless parameters: the capillary number $(C a)$, the ratio of hole diameter to channel height $(b=d / h)$, and the normalized droplet radius $(\bar{R}=R / h)$. The capillary number relates viscous to capillary forces and is defined as $C a=U_{c} \eta_{c} / \sigma$, where $U_{c}=Q_{c} /(w h)$ is the inlet mean flow velocity. In addition, surface wettability also plays an important role in determining the dynamical behavior of a moving droplet in a microchannel due to large surface-to-volume ratio. In order to achieve experiment-matched droplet behavior, it is necessary that the continuous phase preferentially wets the walls [18]. The magnitude of wettability will be described by the contact 
(a)

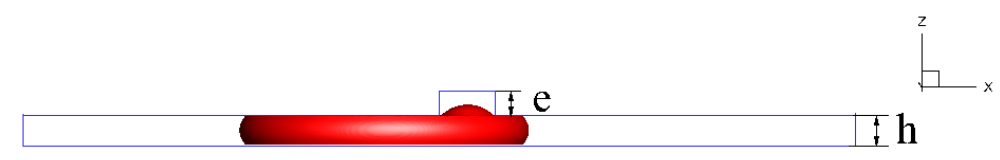

(b)

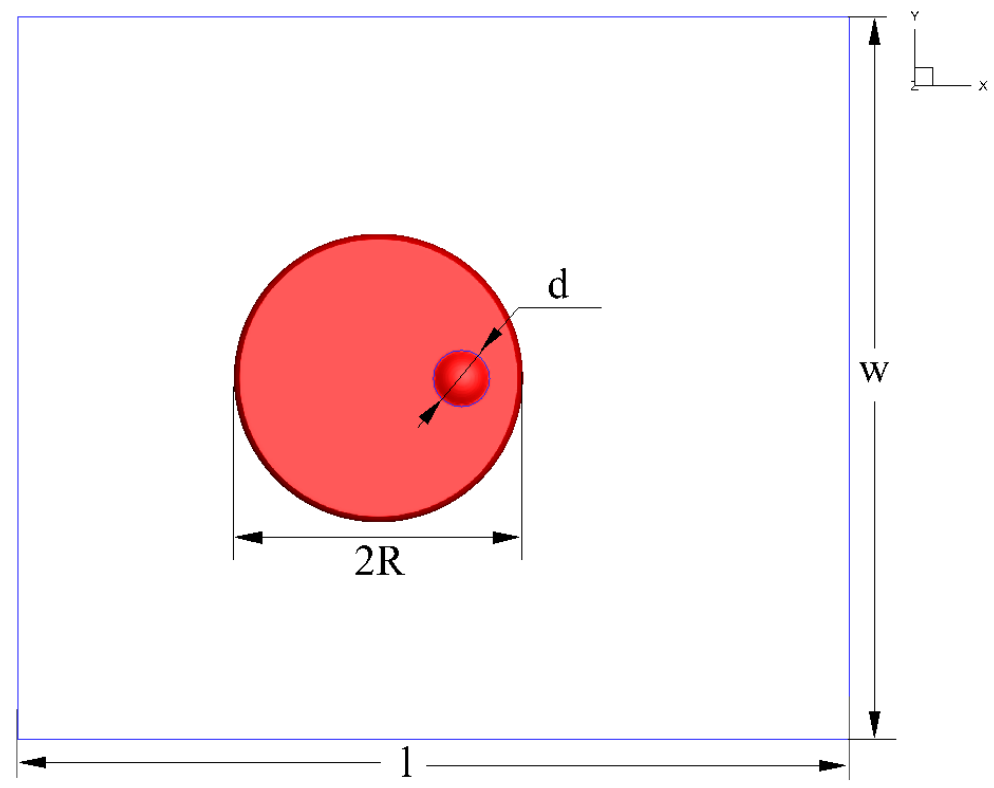

Figure 1: Schematic of a water droplet of radius $R$ subject to an external oil flow in a microchannel that contains a cylindrical anchor of diameter $d$ and of depth $e$ : (a) side view and (b) top view. The microchannel has a height $h$, a length $l$, and a width $w$. The continuous phase oil is injected from the left inlet at a flow rate that is a constant in each case.

angle $\theta$. Here, we will examine the influence of $C a$ and $b$ on the droplet migration for a constant $\bar{R}$, which is fixed at 4 .

Since the phase-field calculation resolves the interface structure, it is computationally too costly for a $3 \mathrm{D}$ simulation to resolve a typical $1 \mathrm{~nm}$ oil-water interface of a microfluidic droplet which is moving slowly. Therefore, 3D phasefield calculation will have to artificially enlarge the interface thickness to simulate droplet behavior. Since the grid resolution may significantly affect the simulation results of the phase-field model, it is important to minimize the numerical error introduced by the grid resolution. We first examine the influence of grid resolution on the numerical results by conducting the simulations with 
Table 1: The LBM simulation parameters for the grid resolutions of $h=20$ and $h=40$ lattices.

\begin{tabular}{cc|ccccc}
\hline \hline LBM simulation parameters & $\rho$ & $\eta$ & $M$ & $\xi$ & $\sigma$ \\
\hline \multirow{2}{*}{ Grid resolution } & $h=20$ & 1 & 0.02 & 0.08 & 1.5 & 0.025 \\
& $h=40$ & 1 & 0.04 & 0.16 & 3.0 & 0.05 \\
\hline
\end{tabular}

the channel heights $h=20,40$ and 80 . It is found that the simulation with $h=80$ is extremely costly to run, far beyond the computing capacity currently available to us. To mitigate this issue, a grid refinement scheme such as the adaptive local grid refinement will be beneficial which can minimize the overall grid number while achieve high accuracy at the interface. Fig.2 shows the simulation results for the channel height of $h=20$ (each grid size corresponds to $2 \mu \mathrm{m}$ ) and $h=40$ lattices (each grid size corresponds to $1 \mu \mathrm{m}$ ) at $C a=3 \times 10^{-4}$, $b=1.8$, and $\theta=160^{\circ}$. To correspond the same physical parameters when the grid resolution is increased from $h=20$ to $h=40$, the LBM simulation parameters, e.g. the density $\rho$, viscosity $\eta$, mobility $M$, interface thickness $\xi$, and the interfacial tension $\sigma$ need to be varied, which are shown in Table 1 for comparison. To match these LBM simulation parameters to their physical values, one needs to choose three reference quantities: a length scale $L_{0}$, a time scale $T_{0}$, and a mass scale $M_{0}$. The values of these reference quantities are $L_{0}=2 \times 10^{-6} \mathrm{~m}, T_{0}=10^{-7} \mathrm{~s}, M_{0}=8 \times 10^{-15} \mathrm{~kg}$ on the coarse grid $(h=20)$, and $L_{0}=1 \times 10^{-6} \mathrm{~m}, T_{0}=5 \times 10^{-8} \mathrm{~s}, M_{0}=1 \times 10^{-15} \mathrm{~kg}$ on the fine grid $(h=40)$. A simulation parameter with dimensions $[\mathrm{m}]^{n 1}[\mathrm{~s}]^{n 2}[\mathrm{~kg}]^{n 3}$ is multiplied by $\left[L_{0}\right]^{n 1}\left[T_{0}\right]^{n 2}\left[M_{0}\right]^{n 3}$ to obtain the physical value. Following this criterion, for example, on the coarse grid, we can obtain the physical value of the density $\rho^{p h y}$ by: $\rho^{p h y}=\rho \frac{M_{0}}{L_{0}^{3}}=\frac{8 \times 10^{-15}}{\left(2 \times 10^{-6}\right)^{3}}=10^{3} \mathrm{~kg} / \mathrm{m}^{3}$, the physical value of the interfacial tension $\sigma^{p h y}$ by: $\sigma^{p h y}=\sigma \frac{M_{0}}{T_{0}^{2}}=0.025 \frac{8 \times 10^{-15}}{\left(10^{-7}\right)^{2}}=0.02 \mathrm{~N} / \mathrm{m}$, and the physical value of the viscosity $\eta^{p h y}$ by: $\eta^{p h y}=\eta \frac{M_{0}}{L_{0} T_{0}}=0.02 \frac{8 \times 10^{-15}}{\left(2 \times 10^{-6}\right) \cdot\left(10^{-7}\right)}=8 \times 10^{-4}$ $\mathrm{Pa}$.s. As illustrated in Fig.2, the grid resolutions with $h=20$ and $h=40$ lattices produce almost the same droplet profiles in the final state, which are 
represented by green dashed lines and blue dash-dot-dot lines, respectively.

In a recent work, Dangla et al. [18] derived a theoretical expression, based on the force balance, for predicting the equilibrium shape of the anchored droplet, i.e.

$$
\frac{\delta r(\varphi)}{R}=15.3 C a \frac{R^{2}}{h^{2}}(1-\varphi \sin \varphi),
$$

where $\varphi$ is the azimuth angle measured counter-clockwise from the positive $x$ axis in the $x y$ plane, and $\delta r(\varphi)$ is the local deviation from the droplet radius $R$. Note that Eq.(28) is valid only when $b \leq 2$ and $\delta r / R \ll 1$. The predicted deformations were found to agree well with the experimental measurements over a broad range of flow conditions [18], and thus Eq.(28) can be used to assess accuracy of the numerical simulations. Fig.2 also presents a comparison between the simulated equilibrium droplet shapes obtained with $h=20$ and 40 lattices and the predicted result from Eq.(28) (represented by red solid line) for $C a=3 \times 10^{-4}, b=1.8$, and $\theta=160^{\circ}$. To quantify the accuracy of the numerical results, we define two relative errors: $E_{x}=\frac{\left|L_{x}-L_{x, a}\right|}{L_{x, a}} \times 100 \%$ and $E_{y}=\frac{\left|L_{y}-L_{y, a}\right|}{L_{y, a}} \times 100 \%$, where $L_{x}$ and $L_{y}$ are the simulated droplet lengths in $x$ direction and $y$-direction respectively, and $L_{x, a}$ and $L_{y, a}$ are their corresponding predicted values from Eq.(28). We find that $E_{x}=1.53 \%$ and $E_{y}=0.85 \%$ on the coarse grid $(h=20)$, close to $E_{x}=1.07 \%$ and $E_{y}=0.58 \%$ on the fine grid $(h=40)$. This suggests that the grid resolution with $h=20$ lattices can provide acceptable numerical accuracy. Thus, the grid resolution with $h=20$ lattices will be used in the subsequent simulations.

The influence of $C a$ is then investigated for $b=1.8$ and $\theta=160^{\circ}$. Different values of $C a$ are achieved by varying $Q_{c}$ solely. As observed by Dangla et al. [18], the droplet motion undergoes two states, depending on the value of $C a$. For $C a \leq 3 \times 10^{-4}$ (the corresponding Reynolds number $R e=\rho Q_{c} /(w \eta) \leq$ $0.375)$, the droplet progresses towards the outlet due to the hydrodynamic drag force from the outer oil flow, and eventually remains anchored to the hole (see Fig.3). The final stationary state of the droplet is a result of the force balance between the force due to surface energy gradient $\left(\vec{F}_{\sigma}\right)$ and the pressure drag 


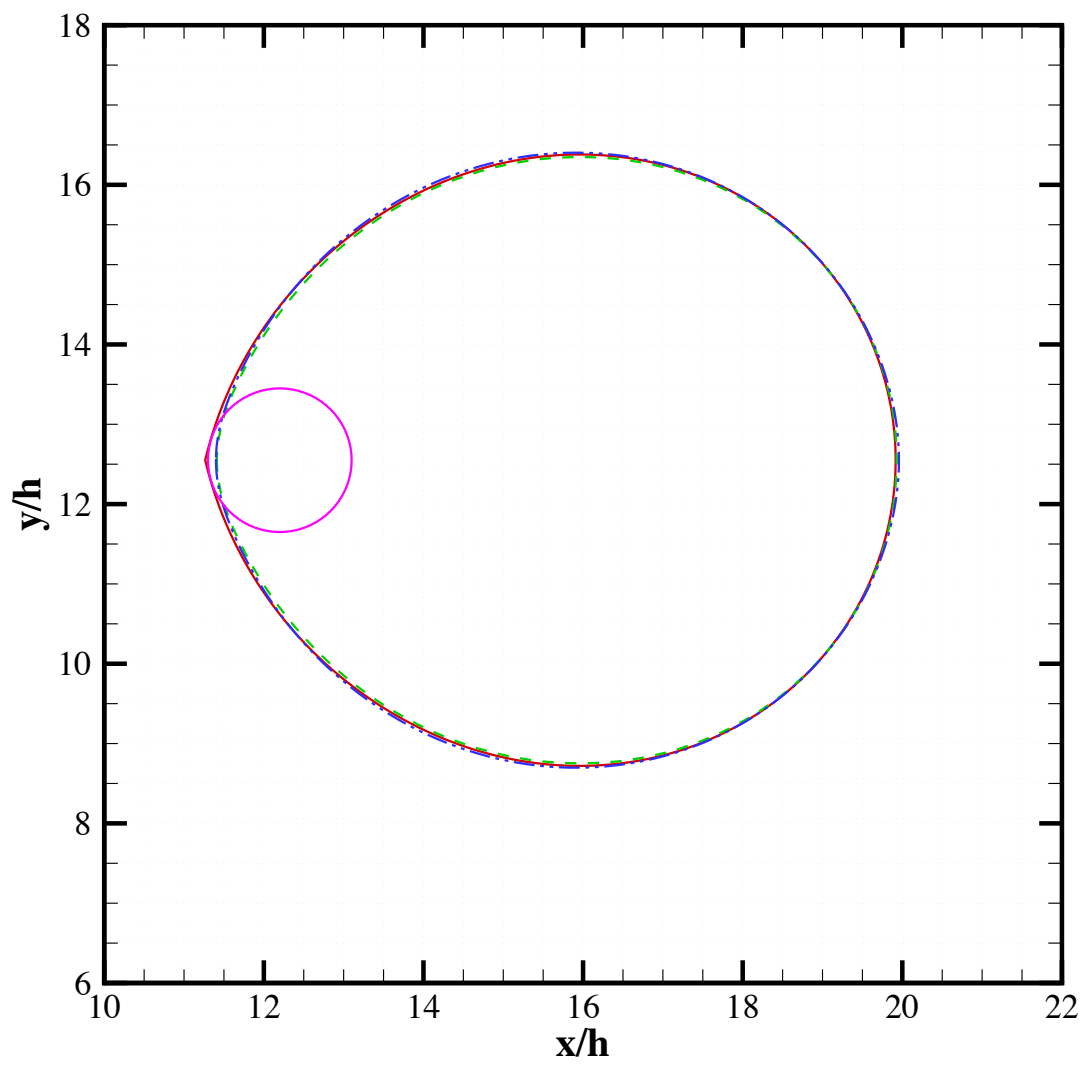

Figure 2: The equilibrium droplet shapes obtained with the grid resolutions of $h=20$ lattices (represented by green dashed lines) and $h=40$ lattices (represented by blue dash-dot-dot lines), and their comparison with the predicted result from Eq.(28) (represented by red solid line) for $C a=3 \times 10^{-4}, b=1.8$, and $\theta=160^{\circ}$. The $x$ and $y$ coordinates are both normalized by the channel height $h$. The location of the hole is indicated by the pink solid lines. 


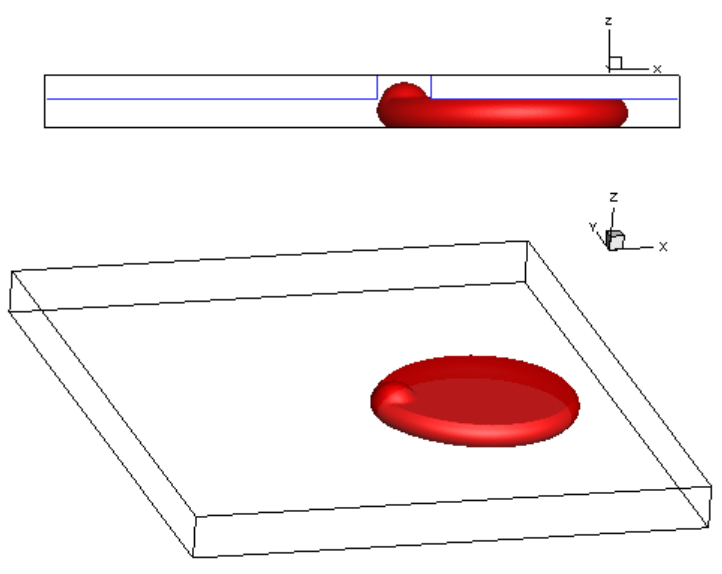

Figure 3: The final shape and position of the droplet for $C a=3 \times 10^{-4}, b=1.8$, and $\theta=160^{\circ}$.

force $\left(\vec{F}_{d}\right)$ [18]. When the capillary number is increased to $5 \times 10^{-4}$ (which corresponds to $R e=0.625$ ), the droplet continuously moves towards the outlet and cannot be completely anchored by the surface energy gradient due to the dominant $\vec{F}_{d}$. Fig. 4 shows the comparison of velocity vectors near the rear of the droplet between $C a=3 \times 10^{-4}$ and $C a=5 \times 10^{-4}$ when the rear of the droplet moves into the hole. We can clearly see that the velocity vectors are negligibly small (which are non-zero because of unphysical spurious currents arising from the model itself) inside the droplet for $C a=3 \times 10^{-4}$, suggesting that the droplet has reached the static state. However, the magnitude of velocity vectors can be comparable to the characteristic flow velocity $U_{c}$ for $C a=5 \times 10^{-4}$. Therefore, it is not surprising that the droplet keeps migrating towards the outlet and eventually moves out of the domain. While passing through the anchor in the present geometry $(b=1.8)$, the droplet only partially enters into the hole and forms a spherical cap, regardless of $C a$. This is consistent with the theoretical model and experimental observations by Dangla et al. [18], in which the parameter $b$ is limited to the case of $b \leq 2$. In addition, we interestingly notice that the rear of the droplet does not strictly touch the junction between the anchor and the top wall of the microchannel when the droplet is trapped 


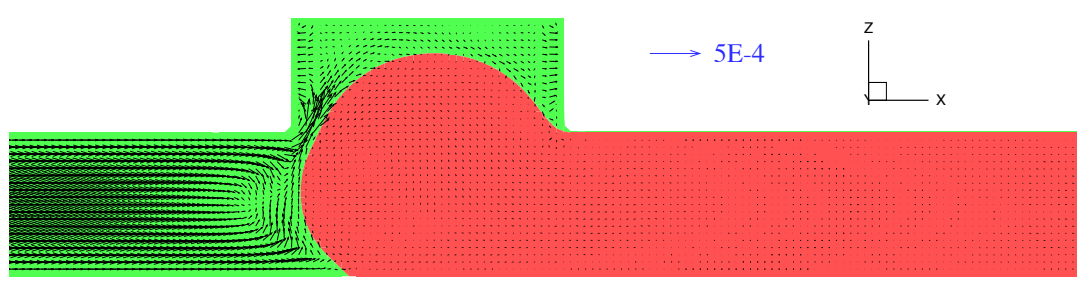

(a)

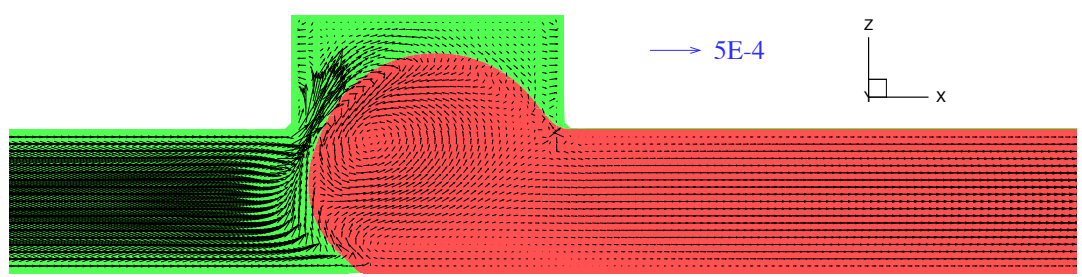

(b)

Figure 4: Velocity vectors near the rear of the droplet for (a) $C a=3 \times 10^{-4}$ and (b) $C a=$ $5 \times 10^{-4}$ when the rear of the droplet moves into the hole. The reference vector $5 \times 10^{-4}$ is shown in blue above the microchannel.

(see Fig.4(a)). It is worthwhile to remark that, in the derivation of Eq.(28), Dangla et al. [18] estimated the surface area of the anchored droplet by assuming the curvature equilibrium between the spherical cap in the hole and the faraway interface, which implies that the rear of the anchored droplet touches the junction, distinct from the present numerical result. The difference might be explained as follows. Physically, there exists small gap between the rear of the anchored droplet and the junction, but the gap is artificially enlarged in our simulations because of the diffuse interface model used, where the interface thickness is artificially enlarged from $1 \mathrm{~nm}$ to several microns. When the actual gap is likely to be on the order of tens or hundreds of nms, it could not be observed in the experiments of Dangla et al. [18], so the assumption of the curvature equilibrium is acceptable. On the other hand, although our numerical simulations artificially enlarge the gap between the rear of the droplet and the junction, this enlargement is found not to affect much the overall accuracy of the numerical results, as demonstrated by the comparison shown in Fig.2. 

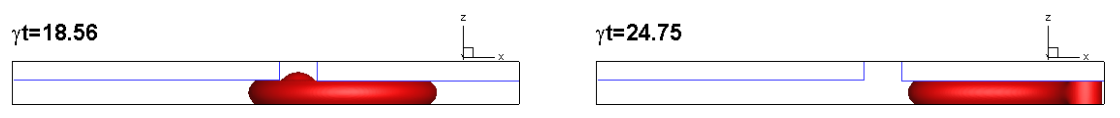

(a) $b=1.5$
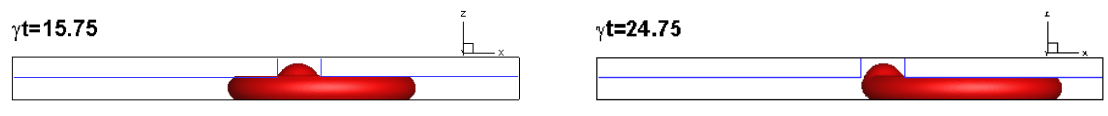

(b) $b=1.8$
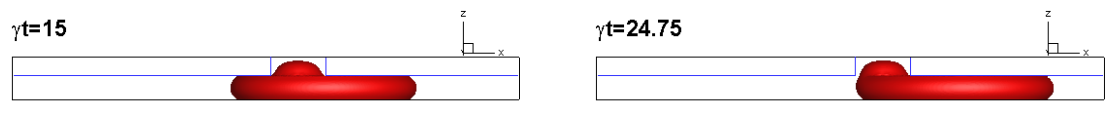

(c) $b=2.2$
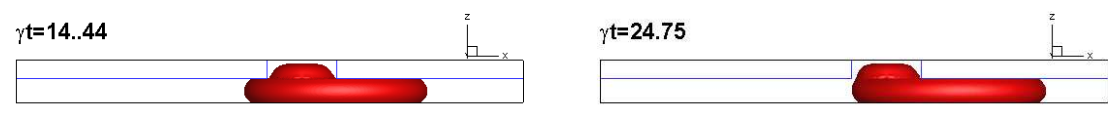

(d) $b=2.8$

Figure 5: Snapshots of droplet shape and position for (a) $b=1.5$, (b) $b=1.8$, (c) $b=2.2$, and (d) $b=2.8$ at $C a=3 \times 10^{-4}$ and $\theta=160^{\circ}$. For each $b$, the left snapshot corresponds to an instant of the droplet body passing through the hole and the right one to the instant of $\gamma t=24.75$. 
Finally, the influence of $b$ is investigated for $C a=3 \times 10^{-4}$ and $\theta=160^{\circ}$. Four different values of $b$ are used in the simulations, i.e., $b=1.5,1.8,2.2$, and 2.8, which are obtained by adjusting the hole diameter while keeping other parameters fixed. Obviously, the used values of $b$ are beyond the limit of $b \leq 2$, thus providing a complement to the study of Dangla et al. [18]. Fig.5 shows the snapshots of droplet shape and position for (a) $b=1.5$, (b) $b=1.8$, (c) $b=2.2$, and (d) $b=2.8$. For each $b$, two snapshots are shown, with the left one corresponding to an instant of the droplet body (all parts except the front and rear) passing through the anchor and the right one to the instant of $\gamma t=24.75$, where $\gamma$ is the shear rate and defined by $\gamma=U_{c} / h$. It can be observed that $b$ can strongly affect the shape of droplet and its motion. As $b$ increases, the amount and height of water droplet that penetrates into the hole increases, resulting in an increase in the anchoring force induced by the surface energy gradients. Thus, the droplet motion undergoes the transition from an unanchored state to an anchored state with increasing $b$ (see the right snapshots in Fig.5). For $b>2$, the spherical cap upwardly abuts against the top wall in the hole (i.e., the droplet height reaches its maximal value), and some continuous phase oil still remains trapped in the hole, which is distinct from the expectation of Dangla et al. [18] that the droplet will fully enter the hole if $b>2$ (note that this expectation is not demonstrated experimentally). Since the droplet interface is confined by the top wall of the hole, it is believed that the hole depth $e$ is an additional important parameter influencing the anchoring behavior of a droplet in the case of $b>2$. When the droplet is eventually anchored, we again observe that its rear interface does not strictly touch the junction for not only $b \leq 2$ but also $b>2$.

To know how the anchor affects the droplet motion, Fig. 6 plots the $x$ coordinate of droplet centroid $\left(x_{d}\right)$ as a function of $\gamma t$ for various $b$ at $C a=$ $3 \times 10^{-4}$ and $\theta=160^{\circ}$, in which $x_{d}$ is normalized by the channel height $h$ and is calculated by

$$
x_{d}(t)=\frac{\int_{V(\phi>0)} x \mathrm{~d} V}{\int_{V(\phi>0)} \mathrm{d} V}=\frac{\sum_{\vec{x}} x(\vec{x}, t) N(\phi(\vec{x}, t))}{\sum_{\vec{x}} N(\phi(\vec{x}, t))}
$$




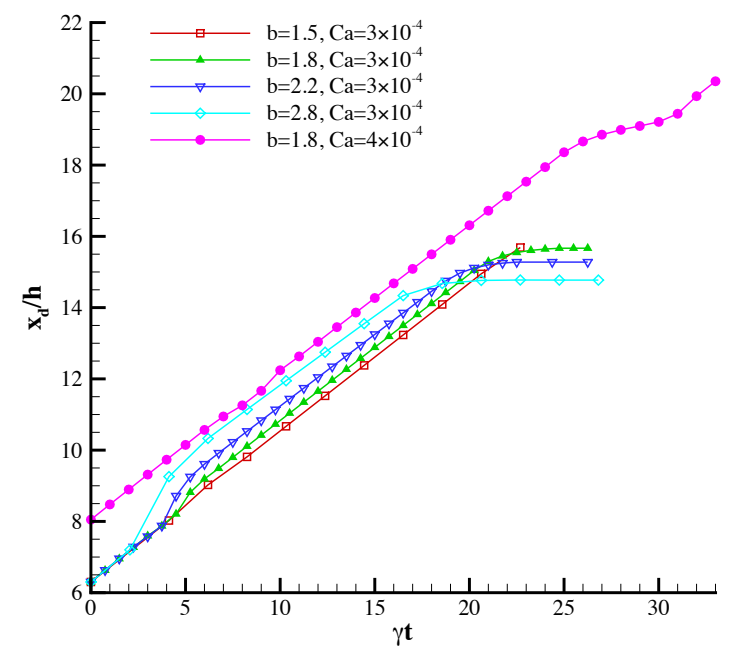

Figure 6: $x$-coordinate of droplet centroid $\left(x_{d}\right)$ as a function of $\gamma t$ for various $b . x_{d}$ is normalized by the channel height $h$. The pink symbols and lines represent the numerical results for $C a=4 \times 10^{-4}$ and $\theta=150^{\circ}$ in a longer computational domain, while all the others are the results for $C a=3 \times 10^{-4}$ and $\theta=160^{\circ}$.

with the function $N(\phi)$ defined by

$$
N(\phi)= \begin{cases}1, & (\phi>0), \\ 0, & (\phi \leq 0) .\end{cases}
$$

By differentiating $x_{d}$ with respect to time, we obtain that, the droplet initially migrates in the channel at a constant velocity for all the values of $b$. When the droplet penetrates into the hole, $x_{d}$ increases in a faster fashion. The increase is more significant for a larger $b$, because a lager $b$ enables more water to penetrate into the hole. As the droplet front moves out of the hole, the droplet migrates again at a constant velocity, although a small portion remains in the hole. The migration velocity is very close to the one when the droplet is far away from the hole (without anchoring force). This suggests that the effect of the anchoring forces can be negligible during the period that the droplet body passes through the hole because the anchoring forces from the surrounding walls of hole 
cancel out due to symmetry. Once the rear of the droplet enters the hole, the symmetry is broken, so the droplet quickly slows down and finally rests with its rear situated at a small gap from the junction for $b \geq 1.8$. This could explain why the anchored interface is always the rear of the droplet rather than other parts, as observed in the previous experiments [18]. For the smallest $b$, i.e., $b=1.5$, we recorded the simulation results every 110, 000 time steps, and found that some of the droplet has moved out of the computational domain if we keep recording the simulation results after $\gamma t=22.69$, which corresponds to the last red square in Fig.6. Once the droplet moves out of the computational domain, we cannot calculate $x_{d}$ correctly. Hence, the simulation results of $b=1.5$ are only displayed until $\gamma t=22.69$ in Fig.6. To reproduce a complete picture of droplet motion in an unanchored state, the channel length is increased to $l=1150 \mu \mathrm{m}$, and the droplet center is changed to $x_{0}=320$ and $y_{0}=250 \mu \mathrm{m}$. The simulation is run with $b=1.8, C a=4 \times 10^{-4}$ and $\theta=150^{\circ}$, and the obtained results are also plotted in Fig.6 (see the pink symbols and lines). As observed in the anchored state, the droplet also undergoes a slow-down process due to the anchoring forces when its rear passes through the hole. However, the anchoring forces are not large enough to pin the droplet completely. Based on these observations, we can conclude that the anchoring forces block the droplet motion only when the droplet rear passes through the hole. Finally, when the droplet is anchored for $b \geq 1.8$, as shown in Fig.6, the rest position is dependent on $b$ : increasing $b$ leads to a decrease in $x_{d}$, which is attributed to the increased volume of the droplet residing in the hole.

\section{Conclusions}

A three-dimensional phase-field LBM is used to simulate the dynamical behavior of a confined droplet subject to an outer viscous flow in a microchannel that contains a cylindrical hole etched into the top surface. The influence of capillary number and hole diameter, which is expressed as the ratio of hole diameter to channel height $(b)$, is investigated. We demonstrate numerically that 
the surface energy gradient induced by the hole can produce an anchoring force to resist the hydrodynamic drag from the outer flow, resulting in the droplet anchored to the hole when the capillary number is below a critical value. When $b$ increases from 1.5 to 2.8 the droplet motion undergoes the transition from an unanchored state to an anchored state. For $b<2$, the droplet partially enters into the hole and forms a spherical cap; whereas for $b>2$, more droplet penetrates into the hole, and the spherical cap abuts upwardly against the top wall of the hole, which makes the hole depth into a crucial parameter. These observations are consistent with the previously reported experiments. However, the droplet does not fully enter the hole for $b>2$, different from the expectation of Dangla et al. [18]. Also, we notice that the rear of the droplet rests at a small distance away from the junction for any $b$ in the anchored state. Finally, regardless of $b$, the droplet always undergoes a slow-down process when its rear passes through the hole.

\section{ACKNOWLEDGEMENTS}

This work is financially supported by the Thousand Youth Talents Program for Distinguished Young Scholars, the National Natural Science Foundation of China (No. 51506168), and the China Postdoctoral Science Foundation (No. 2016M590943). The simulations were performed on the UK national highperformance computer ARCHER which is funded by the UK's Engineering and Physical Sciences Research Council (EPSRC) under grant EP/L00030X/1.

[1] A. R. Wheeler, Putting electrowetting to work, Science 322 (5901) (2008) $539-540$.

[2] J. Gong, C.-J. Kim, All-electronic droplet generation on-chip with realtime feedback control for EWOD digital microfluidics, Lab Chip 8 (2008) 898-906.

[3] J. R. Millman, K. H. Bhatt, B. G. Prevo, O. D. Velev, Anisotropic parti- 
cle synthesis in dielectrophoretically controlled microdroplet reactors, Nat. Mater. 4 (2005) 98-102.

[4] K. Ahn, C. Kerbage, T. P. Hunt, R. M. Westervelt, D. R. Link, D. A. Weitz, Dielectrophoretic manipulation of drops for high-speed microfluidic sorting devices, Appl. Phys. Lett. 88 (2006) 024104.

[5] T. Thorsen, R. W. Roberts, F. H. Arnold, S. R. Quake, Dynamic pattern formation in a vesicle-generating microfluidic device, Phys. Rev. Lett. 86 (2001) 4163-4166.

[6] P. Garstecki, M. J. Fuerstman, H. A. Stone, G. M. Whitesides, Formation of droplets and bubbles in a microfluidic T-junction-scaling and mechanism of break-up, Lab Chip 6 (2006) 437-446.

[7] S. L. Anna, H. C. Mayer, Microscale tipstreaming in a microfluidic flow focusing device, Phys. Fluids 18 (2006) 121512.

[8] C. Baroud, J.-P. Delville, F. Gallaire, R. Wunenburger, Thermocapillary valve for droplet production and sorting, Phys. Rev. E 75 (2007) 046302.

[9] C. Baroud, M. Robert de Saint Vincent, J.-P. Delville, An optical toolbox for total control of droplet microfluidics, Lab Chip 7 (2007) 1029-1033.

[10] H. Liu, Y. Zhang, A. J. Valocchi, Modeling and simulation of thermocapillary flows using lattice Boltzmann method, J. Comput. Phys. 231 (12) (2012) 4433-4453.

[11] H. Liu, A. J. Valocchia, Y. Zhang, Q. Kang, Lattice Boltzmann phase-field modeling of thermocapillary flows in a confined microchannel, J. Comput. Phys. 256 (2014) 334-356.

[12] T. Franke, A. R. Abate, D. A. Weitz, A. Wixforth, Surface acoustic wave (SAW) directed droplet flow in microfluidics for PDMS devices, Lab Chip 9 (2009) 2625-2627. 
[13] M. Okochi, H. Tsuchiya, F. Kumazawa, M. Shikida, H. Honda, Dropletbased gene expression analysis using a device with magnetic force-baseddroplet-handling system, J. Biosci. Bioeng. 109 (2) (2010) 193 - 197.

[14] Y. Zhang, S. Park, K. Liu, J. Tsuan, S. Yang, T.-H. Wang, A surface topography assisted droplet manipulation platform for biomarker detection and pathogen identification, Lab Chip 11 (2011) 398-406.

[15] R. Di Leonardo, G. Ruocco, J. Leach, M. J. Padgett, A. J. Wright, J. M. Girkin, D. R. Burnham, D. McGloin, Parametric resonance of optically trapped aerosols, Phys. Rev. Lett. 99 (1) (2007) 010601-.

[16] D. McGloin, D. R. Burnham, M. D. Summers, D. Rudd, N. Dewar, S. Anand, Optical manipulation of airborne particles: techniques and applications, Faraday Discuss. 137 (2008) 335-350.

[17] P. Abbyad, R. Dangla, A. Alexandrou, C. Baroud, Rails and anchors: guiding and trapping droplet microreactors in two dimensions, Lab Chip 11 (2011) 813-821.

[18] R. Dangla, S. Lee, C. N. Baroud, Trapping microfluidic drops in wells of surface energy, Phys. Rev. Lett. 107 (2011) 124501.

[19] C. Hirt, B. Nichols, Volume of fluid (VOF) method for the dynamics of free boundaries, J. Comput. Phys. 39 (1981) 201-225.

[20] W. J. Rider, D. B. Kothe, Reconstructing volume tracking, J. Comput. Phys. 141 (2) (1998) 112-152.

[21] S. Osher, J. A. Sethian, Fronts propagating with curvature-dependent speed: Algorithms based on Hamilton-Jacobi formulations, J. Comput. Phys. 79 (1) (1988) 12-49.

[22] M. Sussman, E. Fatemi, P. Smereka, S. Osher, An improved level set method for incompressible two-phase flows, Comput. Fluids 27 (1998) 663680. 
[23] H. Liu, Q. Kang, C. R. Leonardi, B. D. Jones, S. Schmieschek, A. Narváez, J. R. Williams, A. J. Valocchi, J. Harting, Multiphase lattice Boltzman$\mathrm{n}$ simulations for porous media applications, Computational Geosciences 20 (4) (2016) 777-805.

[24] H.-Y. Chen, D. Jasnow, J. Viñals, Interface and contact line motion in a two phase fluid under shear flow, Phys. Rev. Lett. 85 (2000) 1686-1689.

[25] A. K. Gunstensen, D. H. Rothman, S. Zaleski, G. Zanetti, Lattice Boltzmann model of immiscible fluids, Phys. Rev. A 43 (8) (1991) 4320-4327.

[26] H. Liu, A. J. Valocchi, Q. Kang, Three-dimensional lattice Boltzmann model for immiscible two-phase flow simulations, Phys. Rev. E 85 (2012) 046309.

[27] X. Shan, H. Chen, Lattice Boltzmann model for simulating flows with multiple phases and components, Phys. Rev. E 47 (3) (1993) 1815-1819.

[28] M. Sbragaglia, R. Benzi, L. Biferale, S. Succi, K. Sugiyama, F. Toschi, Generalized lattice Boltzmann method with multirange pseudopotential, Phys. Rev. E 75 (2007) 026702.

[29] M. R. Swift, E. Orlandini, W. R. Osborn, J. M. Yeomans, Lattice Boltzmann simulations of liquid-gas and binary fluid systems, Phys. Rev. E 54 (5) (1996) 5041-5052.

[30] C. M. Pooley, K. Furtado, Eliminating spurious velocities in the free-energy lattice Boltzmann method, Phys. Rev. E 77 (2008) 046702.

[31] X. He, S. Chen, R. Zhang, A lattice Boltzmann scheme for incompressible multiphase flow and its application in simulation of Rayleigh-Taylor instability, J. Comput. Phys. 152 (2) (1999) 642-663.

[32] A. Fakhari, M. H. Rahimian, Phase-field modeling by the method of lattice Boltzmann equations, Phys. Rev. E 81 (2010) 036707.

[33] T. Lee, L. Liu, Lattice Boltzmann simulations of micron-scale drop impact on dry surfaces, J. Comput. Phys. 229 (20) (2010) 8045-8063. 
[34] J. Y. Shao, C. Shu, H. B. Huang, Y. T. Chew, Free-energy-based lattice Boltzmann model for the simulation of multiphase flows with density contrast, Phys. Rev. E 89 (2014) 033309.

[35] A. J. Briant, J. M. Yeomans, Lattice Boltzmann simulations of contact line motion. II. Binary fluids, Phys. Rev. E 69 (2004) 031603.

[36] X.-D. Niu, T. Munekata, S.-A. Hyodo, K. Suga, An investigation of watergas transport processes in the gas-diffusion-layer of a PEM fuel cell by a multiphase multiple-relaxation-time lattice Boltzmann model, J. Power Sources, 172 (2007) 542-552.

[37] H. Ding, P. D. Spelt, C. Shu, Diffuse interface model for incompressible two-phase flows with large density ratios, J. Comput. Phys. 226 (2007) 2078-2095.

[38] D. Jacqmin, Calculation of two-phase Navier-Stokes flows using phase-field modeling, J. Comput. Phys. 155 (1999) 96-127.

[39] T. Lee, P. F. Fischer, Eliminating parasitic currents in the lattice Boltzmann equation method for nonideal gases, Phys. Rev. E 74 (4) (2006) 046709.

[40] X. He, L.-S. Luo, A priori derivation of the lattice Boltzmann equation, Phys. Rev. E 55 (1997) R6333-R6336. doi:10.1103/PhysRevE.55.R6333.

[41] C. Pan, L.-S. Luo, C. T. Miller, An evaluation of lattice Boltzmann schemes for porous medium flow simulation, Comput. Fluids 35 (2006) 898-909.

[42] C. M. Pooley, H. Kusumaatmaja, J. M. Yeomans, Contact line dynamics in binary lattice Boltzmann simulations, Phys. Rev. E 78 (5) (2008) 056709.

[43] Z. Yu, L.-S. Fan, Multirelaxation-time interaction-potential-based lattice Boltzmann model for two-phase flow, Phys. Rev. E 82 (2010) 046708. 
[44] H. Huang, J.-J. Huang, X.-Y. Lu, Study of immiscible displacements in porous media using a color-gradient-based multiphase lattice Boltzmann method, Comput. Fluids 93 (2014) 164-172.

[45] A. J. C. Ladd, Numerical simulations of particulate suspensions via a discretized Boltzmann equation. (Part I \& II), J. Fluid Mech. 271 (1994) 285-339.

[46] L. Wu, M. Tsutahara, L. S. Kim, M. Ha, Three-dimensional lattice Boltzmann simulations of droplet formation in a cross-junction microchannel, Int. J. Multiphase Flow 34 (2008) 852-864.

[47] Q. Zou, X. He, On pressure and velocity boundary conditions for the lattice Boltzmann BGK model, Phys. Fluids 9 (1997) 1591-1598.

[48] L. Hao, P. Cheng, Lattice Boltzmann simulations of liquid droplet dynamic behavior on a hydrophobic surface of a gas flow channel, J. Power Sources 190 (2009) $435-446$.

[49] H. Liu, Y. Zhang, Lattice Boltzmann simulation of droplet generation in a microfluidic cross-junction, Commun. Comput. Phys. 9 (5) (2011) 12351256. 